\title{
SATISFACCIÓN CON LA VIDA EN UN GRUPO DE MIGRANTES GUATEMALTECOS DEPORTADOS DESDE LOS ESTADOS UNIDOS DE AMÉRICA
}

\author{
ALEJANDRO J. MENA ${ }^{1}$ \\ MARIO ANÍBAL HERNÁNDEZ ${ }^{2}$ \\ HÉCTOR BARRIOS 3
}

Fecha de recepción: noviembre de 2018

Fecha de aceptación y versión definitiva: enero de 2019

\begin{abstract}
RESUMEN: El estudio tuvo como objetivo identificar el nivel de satisfacción con la vida en un grupo de migrantes guatemaltecos retornados desde los EE. UU. a Guatemala. Participaron 110 personas (Hombres 86,50\% y 13,50\% mujeres). Se utilizó una escala de datos sociodemográficos y la escala de satisfacción con la vida (Diener et al., 1985). Los resultados indican que 74,80\% de los participantes manifiestan estar satisfechos con su vida a pesar de haber sido deportados desde los EE. UU. Se realizó una serie de correlaciones entre las variables sociodemográficas y el nivel de satisfacción con la vida y se evidenció una correlación negativa entre satisfacción con la vida y el número de intentos por llegar a Estados Unidos ( $r=-0.318$; $p<0.01)$.
\end{abstract}

PALABRAS CLAVE: satisfacción con la vida; migraciones; Guatemala.

\section{Satisfaction with life in a group of Guatemalan migrants deported from the United States of America}

ABSTRACT: The study aimed to identify the level of satisfaction with life in a group of Guatemalan migrants returned from the US to Guatemala. 110 people participated $(86,50 \%$ men and 13,50\% women). We used a scale of sociodemographic data and the Life Satisfaction Scale (Diener et al.,1985). The results indicate that $74,80 \%$ of the participants are satisfied with their lives despite having been

\footnotetext{
1 Universidad Rafael Landívar de Guatemala. Correo electrónico: jandromb@hotmail.com.

2 Universidad Rafael Landívar de Guatemala. Correo electrónico: mahernandezgt@gmail.com

3 Universidad Rafael Landívar de Guatemala. Correo electrónico:
} hbpc3@hotmail.com 
deported from the US. A series of correlations was made between the sociodemographic variables and the level of satisfaction with life, thus, a negative correlation between satisfaction with life and the number of attempts to reach the United States was evidenced $(r=-0.318 ; p<0.01)$.

KEY WORDS: life satisfaction; migrations; Guatemala.

\section{INTRODUCCIÓN}

La migración en su concepción más amplia implica una serie de fenómenos que atraviesan las esferas social, personal, económica y laboral (Bermúdez \& López, 2019). Aunque los motivos de las migraciones son multicausales y dependen de diversos y complejos factores, desde una visión general del fenómeno, puede decirse que ellas obedecen a la necesidad de encontrar mejores condiciones de vida que y evitar riesgos y peligros (Adger, et al., 2015; Aguilar, Taylor, \& Castellanos, 2016). Dichas características han llamado la atención de diversos investigadores e instituciones que buscan dar respuesta a esta problemática global. El fenómeno de la migración es comúnmente analizado desde áreas del conocimiento como la economía, la sociología o la ciencia política, cada una de ellas, desde sus particulares énfasis e interpretaciones (Sosa \& Zubieta, 2012). Mientras tanto, son pocos los estudios que han buscado evaluar componentes cognitivos o afectivos, propios de la esfera psicológica de quienes por diversos factores han decidido dejar su tierra de origen.

\section{CONDICIONES DE VIDA EN GUATEMALA Y SATISFACCIÓN CON LA VIDA}

Aunque el fenómeno de las migraciones es una problemática presente en diversos países alrededor del mundo, dicho fenómeno adquiere características particulares en el contexto denominado triángulo norte de América Central (Guatemala, El Salvador y Honduras). De esa manera, los países que conforman el triángulo norte suelen expulsar a sus ciudadanos por múltiples razones dentro de las que se puede mencionar la falta de oportunidades laborales, escolares, económicas y falta de seguridad.

Aunque las causas de la migración son diversas y complejas, un aspecto que merece especial atención y que ha cobrado especial relevancia, es la 
seguridad en esos países. De acuerdo con la Oficina de la Naciones Unidas Contra la Droga y el Delito (UNODC, 2013) En Guatemala, el índice de muertes violentas entre 2003 y 2012 fue de entre 30 y 40 muertes por cada 100,000 habitantes (UNODC, 2013). De esa manera, puede decirse que la migración para la mayoría de guatemaltecos no es un acto de libertad sino una estrategia de sobrevivencia ante las escasas oportunidades laborales, de educación, progreso y ante la falta de seguridad (López \& Rivera, 2014).

Paralelamente con lo anterior, de acuerdo con el Informe Nacional de Desarrollo Humano, del Programa de las Naciones Unidas para el Desarrollo (PNUD, 2016) los índices de desarrollo humano en Guatemala son inversos al impulso que ha tenido la economía guatemalteca en los últimos años. En ese sentido cabe resaltar que a pesar de evidenciarse un incremento en la calidad de vida de los guatemaltecos entre el 2000 y 2014, dicho incremento no ha sido constante, especialmente durante el primer lustro del nuevo milenio. Como se menciona en dicho informe, la expansión de la riqueza económica no necesariamente ha significado una mejora en la vida de las personas en Guatemala, de hecho, tal expansión parece haber incrementado la brecha entre ricos y pobres, situación que se hace evidente en el deterioro ecológico, social y donde el aumento de la migración hacia los Estados Unidos de América es un síntoma más.

Derivado de las sensibles carencias que atraviesa gran parte de la población guatemalteca, el flujo de migrantes (incluidos niños) ha sido constante durante los últimos años, de manera que según el Programa de las Naciones Unidas para el Desarrollo PNUD (2016), en Estados Unidos de América habitan aproximadamente 881.191 ciudadanos guatemaltecos. Otro dato interesante derivado del informe realizado por Naciones Unidas es que 45,2\% de los guatemaltecos deja de consumir, como estrategia para cubrir gastos cuando no cuenta con dinero, mientras que un 35,7\% acude a préstamos. Tales datos reflejan el contexto en el que vive un alto porcentaje de la población en Guatemala, contexto que en muchas ocasiones deriva en la búsqueda de nuevas y mejores oportunidades. Por otro lado, para la Organización Mundial de la Salud (OMS, 2003) el bienestar responde no únicamente a dimensiones vinculadas con la salud física sino también mental y social, de tal forma que dichos factores se encuentran enmarcados dentro de una interacción armónica con el ambiente. Lo anterior pone en evidencia que las razones para migrar suelen ser diversas, se puede decir que:

Las dinámicas migratorias en el país son históricas y complejas. Guatemala es un país de origen, tránsito, destino y (cada día más) de deportación de migrantes. Dadas las enormes diferencias entre guatemaltecos, en el país conviven personas con capacidad para movilizarse y migrar libremente en busca de mejores 
oportunidades de vida y personas que migran para sobrevivir. Dentro de este continuum se encuentran infinidad de personas que con razones distintas para migrar han transformado las relaciones sociales, económicas y culturales del país (López Robles y Rivera 2013, p. 1).

Como fue mencionado previamente, los motivos para migrar son diversos y aunque las motivaciones económicas y de seguridad explican en gran medida las constantes migraciones, éstas no son las únicas razones. De acuerdo con el informe El salto al norte del Fondo de las Naciones Unidas para la Infancia UNICEF (2011) después del factor económico se encuentra la intención de reunirse con la familia, conflictos familiares en el país de origen, problemas con las autoridades y desastres naturales. De manera general y con el objetivo de dar una visión que complemente estudios sobre migración, es importante tomar en cuenta que todo proceso de migración va acompañado de diversos elementos psicológicos como el sentimiento de pérdida, luto y ansiedad ante un entorno físico y cultural diferente y la búsqueda de una vida con sentido, entre otros (Peterson, Park, \& Seligman, 2005). Tal como lo plantean Vallejo-Martín, Jimenez y Del Pilar (2016) «los flujos migratorios son fenómenos sociales que tienen importantes repercusiones tanto a nivel individual como grupal (comunidad de origen y comunidad de destino)»(p. 17). Esta situación parece estar relacionada con la búsqueda de mejores condiciones de vida fuera del país, considerando la carencia de oportunidades, la desigualdad y la exclusión a la que un alto porcentaje de guatemaltecos se ve expuesto. Es por ello que se considera importante que, de la mano de propuestas para atender las causas estructurales de la migración masiva de guatemaltecos, se busque fomentar también espacios de intercambio y conocimiento respecto de la realidad que la población migrante vive.

Así, de la mano de causas eminentemente económicas, políticas o sociales, la literatura respecto de variables psicológicas que acompañan los procesos de migración tanto nacional como internacional es escasa, de hecho, dicha literatura se perfila como un área de estudios sobre migraciones de alta relevancia (De Jong, Chamratrithirong, \& Tran, 2002). Por ello, se considera importante conocer acerca aspectos vinculados con el interés por tener diversos aspectos de la vida integrados en la población migrante. Es así como Shin y Johnson (1978) definen el concepto «satisfacción con la vida» como el juicio general que las personas realizan respecto de su propia vida. Por su parte, Diener (1984) conceptualizó el término buscando ir más allá de las condiciones necesarias para una buena vida (salud, techo, educación, etc.). Propuso un enfoque psicosocial integral, el cual incluye elementos 
vinculados con la satisfacción personal y la felicidad percibida respecto de la propia vida (Perez-Escoda \& Alegre, 2014 y Castro-Solano, 2011).

Si bien es cierto que la satisfacción con la vida es un constructo ampliamente estudiado, principalmente en estudios anglosajones, el mismo es aún poco abordado en el contexto latinoamericano. Paralelamente, no sólo en Guatemala se encuentran pocos estudios que aborden la complejidad de variables psicológicas (como satisfacción con la vida) en la población migrante, dicha carencia se hace evidente también en América Latina. Uno de esos pocos estudios fue realizado en Colombia por Murillo y Molero (2016), quienes abordan el concepto al buscar identificar predictores de la satisfacción en variables psicológicas como la autoestima, pero también en variables vinculadas con logros económicos o materiales como el bienestar material percibido. De esa manera, a través de un análisis de regresión, refieren que las variables de autoestima y bienestar material percibido explican, en conjunto con otras variables, el $23 \%$ de la varianza, es decir que dichas variables muestran un peso importante en la satisfacción con la vida de los participantes del estudio.

Considerando que el perfil de los migrantes no es homogéneo alrededor del mundo, es importante que desde América Latina se desarrollen estudios de investigación y propuestas que aborden la problemática de los migrantes desde perspectivas amplias, plurales e incluyentes, las cuales puedan indagar en la realidad de dicha población más allá de elementos estrictamente económicos; por lo que el presente estudio pretende identificar el nivel de satisfacción con la vida en migrantes deportados, buscando así contribuir con la población migrante desde el campo de la psicología.

\section{MÉTODO}

El presente es un estudio con enfoque cuantitativo, diseño no experimental-transversal y con alcance descriptivo. En ese sentido, el presente trabajo buscó identificar y cuantificar la percepción de un grupo de migrantes respecto de la variable satisfacción con la vida.

Participaron en la investigación 110 personas recientemente deportadas vía aérea desde Los Estados Unidos de América; 86,50\% de la muestra de participantes fueron hombres y 13,50\% fueron mujeres. La selección de los participantes se realizó por medio de un muestreo no probabilístico por conveniencia. Luego de solicitar su participación, los entrevistados llenaron 
el consentimiento informado. La aplicación de los instrumentos se realizó en los meses de julio, agosto y septiembre de 2016.

Para medir la variable satisfacción con la vida se aplicó la escala de satisfacción con la vida de Diener et al. (1985) adaptada al español por Cabañero et al. (2004). El Alfa de Cronbach para la versión original fue de 0,82, mientras que para la presente muestra fue de $\alpha 0,61$. Dichos resultados indican que la prueba utilizada cuenta con propiedades psicométricas aceptables, por lo que se puede decir que la misma cuenta con sustento teórico y psicométrico para medir dicho constructo. Adicionalmente, se administró una hoja de datos sociodemográficos.

\section{RESULTADOS}

\subsection{RESUlTADOS SOCIODEMOGRÁFICOS}

Respecto de los resultados relacionados con las características sociodemográficas de la muestra, pudo observarse, tal como era previsible, que la mayoría de los migrantes deportados desde los Estados Unidos de América eran hombres $(86,5 \%)$ mientras que una minoría estuvo conformada por mujeres $(12,6 \%)$.

De manera previsible, otra de las características sociodemográficas del grupo de migrantes es que la mayoría de ellos eran solteros (55\%) mientras que poco menos de la mitad era casado, en unión libre o divorciados $(44,1 \%)$. Como era de esperarse, la mayoría de los migrantes evaluados eran adultos jóvenes, lo que abona a la hipótesis que refleja carencia de oportunidades para las juventudes en Guatemala, considerando que el 55,5\% de los participantes tenía entre 18 y 25 años.

Probablemente uno de los rasgos más sobresalientes de los migrantes evaluados responde a los motivos por los cuales decidieron dejar el país y buscar mejores oportunidades en el extranjero. Así, por ejemplo, el 66\% indicó que el principal motivo por el cual abandonó el país responde a motivos económicos y a la búsqueda de oportunidades que brindasen un mejor futuro para ellos y sus familias. Al comparar otros motivos por los cuales decidieron salir del país, se pudo observar un número considerable de personas que reportaron que debieron abandonar el país debido a amenazas a su integridad física o persecución (3\%). Asimismo, reunirse con familiares residentes en los Estados Unidos de América fue otra de las razones que los impulsó a salir de Guatemala (15,3\%). 


\subsection{EsCALA DE SATISFACCIÓN CON LA VIDA}

De acuerdo con los resultados obtenidos en la escala de satisfacción con la vida, para el enunciado «En la mayoría de casos mi vida es como quiero», el 52\% de la muestra respondió de manera afirmativa, es decir que sólo cerca de la mitad tiene una actitud favorable hacia la mayoría de situaciones dadas en su vida. Por otro lado, el $25 \%$ considera que no, es decir consideran que existan condiciones que los hacen afirmar que su vida no es como ellos desean.

Por su parte ante el ítem «Las circunstancias de mi vida son buenas» contrario a lo esperado (debido a la realidad que atraviesa la mayoría de migrantes) los resultados muestran que el 63\% refieren estar de acuerdo o totalmente de acuerdo con el enunciado. Los resultados sugieren que desde la óptica de los participantes hay razones para pensar que los eventos vividos son favorables.

Luego de analizar el segundo enunciado se verificó lo expresado en la frase "Estoy satisfecho con mi vida». Nuevamente de manera inesperada, los resultados muestran estar de acuerdo con el mencionado ítem 74,8\%.

Posteriormente se analizó el ítem «Hasta ahora he conseguido de la vida cosas importantes» dicho ítem mostró un comportamiento reducido respecto de los dos ítems analizados anteriormente ya que sólo poco más de la mitad (54\%) indicó estar de acuerdo o totalmente de acuerdo con la expresión. Dicho resultado sugiere, en principio, que los resultados alcanzados por buena parte de la muestra analizada son insuficientes o exiguos.

Por último, la frase «Si pudiera vivir mi vida otra vez, no cambiaría nada» es reveladora, considerando que es la única que muestra dirección predominantemente negativa. En este caso el 45,9\% de los guatemaltecos participantes del estudio indicaron estar en desacuerdo o muy en desacuerdo con la frase. De manera particular los resultados presentados por los participantes hacen notorio que el hecho de haber dejado el país refleja un profundo deseo de cambiar sus condiciones de vida. 
TABLA 1. CORRELACIÓN ENTRE SATISFACCIÓN CON LA VIDA E INTENTOS PARA LLEGAR A LOS EE. UU. E INTENSIDAD CON LA QUE DESEA INTENTAR VOLVER A LOS EE. UU.

\begin{tabular}{|c|c|c|c|}
\hline & Correlaciones & & \\
\hline & & $\begin{array}{c}\text { Intentos } \\
\text { para llegar a } \\
\text { EE. UU. }\end{array}$ & $\begin{array}{l}\text { Satisfacción } \\
\text { con la vida }\end{array}$ \\
\hline \multirow[t]{3}{*}{$\begin{array}{l}\text { Intentos Para llegar a } \\
\text { EE. UU. }\end{array}$} & $\begin{array}{l}\text { Correlación de } \\
\text { Pearson }\end{array}$ & 1 & $-.318 * *$ \\
\hline & Sig. (bilateral) & & 0.001 \\
\hline & $\mathrm{N}$ & 110 & 110 \\
\hline
\end{tabular}

Fuente: elaboración propia (2017).

Además de los datos proporcionados por la escala de satisfacción con la vida se utilizó la $r$ de Pearson para identificar potenciales asociaciones entre las variables sociodemográficas y el resultado global en dicha escala. De esa manera se procedió a transformar el resultado total en medidas de tendencia central (Media = M y Desviación Standard = DE).

De esa forma los resultados revelan una media de $\mathrm{M}=73.7$ y una Desviación estándar de $\mathrm{DE}=26.3$. Al analizar dichos resultados se observa que en términos generales el nivel de satisfacción con la vida es moderadamente alto. Lo anterior sugiere que, a pesar de las circunstancias, los participantes consideran que su vida es buena.

Luego de identificar los resultados a través de los estadísticos descriptivos, se procedió a realizar análisis propios de la estadística inferencial. En este caso se procedió a revisar el tipo de distribución de la muestra, dando como resultado que dicha muestra se encontraba distribuida normalmente. Una vez considerado el criterio anterior, se realizó el análisis de correlación de Pearson $(r)$ entre satisfacción con la vida y diversos datos sociodemográficos recabados.

En la tabla 1 se muestran los resultados de las correlaciones $r$ de Pearson. Contrario a lo que se pensaba, no existe correlación estadísticamente significativa entre satisfacción con la vida y la mayoría de datos sociodemográficos. Únicamente se observó correlación significativa (inversa o negativa) entre la cantidad de intentos para llegar a los Estados Unidos de América y satisfacción con la vida, de esa forma el valor de $p$ sugiere una relación estrecha entre ambas variables $(r=-0.38, \mathrm{P}<0.05)$. Ese resultado soporta 
parcialmente la hipótesis que indicaba que cuanto mayor es la satisfacción con la vida, menor es el interés por abandonar el país de origen.

\section{DISCUSIÓN}

El objetivo del estudio fue identificar los niveles de satisfacción con la vida en un grupo de migrantes recién retornados desde los Estados Unidos. De alguna manera distinto de lo esperado, los niveles de satisfacción con la vida son, en términos generales, favorables en la presente muestra de participantes. Tales resultados parecen en principio sorprendentes, considerando que la mayoría de participantes reportaron las dificultades económicas como principal motivo para buscar oportunidades en los Estados Unidos de América. Así, estudios como el de Murillo y Molero (2016) en Colombia coincide con el presente estudio, tomando en cuenta que variables vinculadas con la autoestima son predictoras de una alta satisfacción con la vida.

Por otro lado, en la presente pesquisa se hizo evidente que, aunque en términos generales los participantes mostraron niveles medios y altos en satisfacción con la vida, dichos resultados merecen ser analizados desde diversos ángulos, considerando que la hipótesis inicial del estudio suponía que una de las consecuencias de una deportación incluiría deterioro en la satisfacción con la vida, sin embargo, los resultados muestran una dirección favorable. Tales resultados pueden interpretarse a la luz de los elementos que componen la satisfacción con la vida, uno de ellos es el hecho de alcanzar logros personales. De esa forma es razonable especular que el llegar a los Estados Unidos y haber podido experimentar el bienestar material y nutrirse de los valores y estilo de vida de ese país sea considerado como un elemento que proporciona profunda satisfacción. En líneas generales dichos resultados recalcan que el haber llegado al lugar de destino y obtener un trabajo representa «no sólo una fuente de ingresos sino también una fuente de salud física, mental y prestigio» (Gamero, como se citó en Vallejo-Martin, Jimenez \& Del Pilar, 2016, p. 18).

En contraposición con lo expresado anteriormente, el enunciado " $\mathrm{Si}$ pudiera vivir mi vida otra vez, no cambiaría nada» indica que más de la mitad de participantes si cambiaría eventos y situaciones en su vida; dicha respuesta puede potencialmente ser explicada a la luz de las condiciones de vida que la mayoría de participantes reportó, ya que 81,1\% indicó que los principales motivos por los que migraron hacia los Estados Unidos de América fueron de tipo económico y familiar. Al analizar la idea de poder 
cambiar su vida desde las condiciones externas debe considerarse los obstáculos y desafíos que van más allá de las condiciones económicas, tomando en cuenta que en muchas ocasiones los migrantes son víctimas de explotación laboral, el racismo y al igual que en Guatemala, de la desprotección y hasta persecución por parte del Estado. Por su parte desde la esfera psicológica, el resultado recientemente descrito puede reflejar las complejidades a las que los migrantes se ven expuestos en su travesía. Es razonable pensar que el abandono del hogar, la soledad, el miedo y la lucha por sobrevivir son condiciones que invariablemente influyen en la visión que se tiene sobre la propia vida.

Es importante resaltar que, aunque el constructo autoestima no fue medido en la presente investigación, se considera que su medición en futuros estudios podría aportar mayor información para explicar las razones por las que a pesar de las condiciones de vida, la mayoría de participantes reporta niveles medios y altos para la variable satisfacción vital. Lo anterior es confirmado en estudios como el de Castro-Solano (2011) quien sostiene que la autoestima es un importante predictor de la satisfacción con la vida. En ese mismo orden de ideas el estudio realizado por Murillo y Molero (2015) pone en evidencia esa posible explicación, considerando que el resultado del estudio realizado con migrantes colombianos reportó correlación significativa entre satisfacción con la vida y autoestima $(\mathrm{r}=0.44, \mathrm{P}<0.05)$.

Tal como lo mencionan los autores previamente citados, las personas suelen estar satisfechas porque experimentan muchas situaciones o momentos gratos derivados principalmente de tres fuentes: el haber recibido apoyo social, haberse sentido satisfechos laboralmente y sentirse satisfechos consigo mismos. En concordancia con lo anterior, los resultados en el presente estudio muestran que un alto porcentaje de participantes migraron hacia los Estados Unidos de América con el objetivo de reunirse con sus familiares, dicho resultado coincide con la literatura, la cual apunta consistentemente que la soledad es un elemento negativamente asociado con la satisfacción con la vida (Diener et al., 1985; Neto 1995; Perez-Escoda y Alegre, 2014). De manera previsible los resultados encontrados en este estudio coinciden con dichas fuentes de satisfacción planteadas por Diener et al. (1985) considerando que el 100\% de los participantes reportaron tanto haber sido contratados en los Estados Unidos de América como haber recibido apoyo de familiares y amigos durante su estancia en dicho país. Evidentemente el simple hecho de laborar en los Estados Unidos no es sinónimo de satisfacción, sin embargo, esa situación puede ser reflejo de una percepción de bienestar subjetivo por parte de los evaluados. 
$\mathrm{Al}$ analizar los resultados encontrados en el presente estudio y con el fin de entender los motivos de por qué se observa altos niveles de satisfacción con la vida, pudo identificarse que la literatura parece ser consistente al identificar el elemento tiempo de residencia en el país destino como un componente de notable importancia. De esa forma, cuando se mide factores psicológicos como satisfacción con la vida, parece existir una tendencia a expresar percepciones $\mathrm{u}$ opiniones favorables, principalmente durante los primeros meses o años después de la llegada al país receptor. Sin embargo, con el pasar del tiempo dicha satisfacción suele mostrar una disminución al asimilar los valores de la comunidad local, principalmente en términos de exigencia y expectativas. Otra posible explicación para esa modificación en la percepción de satisfacción con la vida, es el hecho que, en los meses y años iniciales, aún con ingresos inferiores a la media del país receptor, existe la percepción de mejoría respecto de las condiciones en el contexto del país de origen; situación que evoluciona al profundizarse los vínculos en el país de llegada y por lo tanto el grupo de referencia es paulatinamente transferido al nuevo país de residencia (Jackson et al., 2019; Olgiati, Calvo, \& Berkman, 2013; Piore, 1979).

Por último, es importante señalar algunas de las limitaciones a las que el presente estudio estuvo sujeto. La primera es el hecho de haber aplicado un instrumento de autoinforme, que, a pesar de haber sido respondido con tiempo suficiente, pudo presumiblemente incrementar artificialmente los valores observados, debido a la natural deseabilidad social. En segundo lugar, el nivel educativo de algunos participantes pudo poner en entredicho la completa comprensión de los enunciados, y aunque en esos casos se contó con el apoyo de un evaluador capacitado, es un elemento que se debe resaltar. Además, la condición de indocumentados del total de los participantes pudo probablemente orientar a los participantes a responder de una manera que evitara por cualquier motivo ser identificados. Finalmente, por la naturaleza propia del estudio, fue necesario utilizar una muestra no probabilística, lo que impide la generalización de los resultados aquí presentados. Debido a las razones expuestas con anterioridad, se hace necesaria la realización de nuevos estudios que permitan explicar y comprender de manera más amplia diversos constructos vinculados con la psicología en una población que ha sido tradicionalmente excluida y marginada. 


\section{CONCLUSIONES}

Tomando en cuenta las dificultades que el contexto de recolección de datos representó, pudo identificarse que tanto el hecho de haber llegado a los Estados Unidos de América, como el hecho de haber resistido a las amenazas del trayecto, son elementos que podrían mediar la percepción sobre satisfacción con la vida. Consideramos importante resaltar que, a pesar de las limitaciones del presente estudio, uno de los aportes más importantes del presente estudio es el hecho de dar visibilidad a una realidad que carece de interés por parte de las autoridades en el contexto centroamericano y específicamente guatemalteco. De esa manera y con base en los objetivos de la misma, pudo identificarse lo siguiente:

- Cerca de la mitad de los participantes afirman estar satisfechos con su vida.

- Más de la mitad de los participantes indicó que las circunstancias de su vida en términos generales, son favorables.

- La frase «Si pudiera vivir mi vida otra vez, no cambiaría nada» fue la única que mostró una tendencia predominantemente negativa.

- Existe correlación moderada, significativa y negativa entre la cantidad de intentos por llegar a los EE. UU. y el nivel de satisfacción con la vida.

- En términos generales el perfil de participantes (recién deportados a Guatemala) estuvo conformado por hombres solteros jóvenes.

- Se hace necesario ampliar las variables de estudio más allá de la satisfacción con la vida considerando la importancia social del fenómeno global de las migraciones.

- Considerando el reducido recorte del presente estudio, se considera necesario ampliar estudios similares a contextos más allá de la realidad guatemalteca.

\section{REFERENCIAS}

Adger, W. N., Arnell, N. W., Black, R., Dercon, S., Geddes, A., y Thomas, D. S. (2015). Focus on environmental risks and migration: causes and consequences. Environmental Research Letters, 10(6), 060201

Aguilar S., Taylor, M., \& Castellanos, E. (2016) Agriculture, Land Tenure and International Migration in Rural Guatemala. Journal of Agrarian Change, 16(1), 123-144. https://doi.org/10.1111/joac.12091. 
Bermudez, R., y López, L. (2019). Trayectorias laborales de migrantes calificados retornados a la ciudad de Cali, Colombia. Migraciones. Publicación del Instituto Universitario de Estudios sobre Migraciones, (46), 35-61.

Cabañero, J. M., Martínez, M. R., Cabrero, J. C., Orts, M. I., Reig, A., y Tosal, B. (2004). Fiabilidad y validez de la escala de Satisfacción con la Vida de Diener en una muestra de mujeres embarazadas y puérperas. Psicothema, 16(3), 448-455.

Castro Solano, A. (2011). Acculturation strategies and psychological and sociocultural adaptation of foreign students in Argentina. Interdisciplinaria, 28(1), 115-130.

For Better, For Worse: Life Satisfaction Consequences of Migration. International Migration Review, 36(3), 838-863.

Diener, E., Emmons, R., Larsen, R. J., y Griffin, S. (1985). The satisfaction with life scale. Journal of Personality Assessment, 49, 71-75. Disponible en: http://s.psych. uiuc.edu/ ediener/SWLS.html.

Fondo de las Naciones Unidas para la Infancia, UNICEF (2011). El salto al norte: Violencia,

inseguridad e impunidad del fenómeno migratorio en Guatemala. Recuperado de: https://www.wikiguate.com.gt/w/images/8/8b/El_Salto_al_Norte.pdf.

Jackson, Y., Courvoisier, D. S., Duvoisin, A., Ferro-Luzzi, G., Bodenmann, P., Chauvin, P., y Burton-Jeangros, C. (2019). Impact of legal status change on undocumented migrants' health and well-being (Parchemins): protocol of a 4-year, prospective, mixed-methods study. BMJ open, 9(5), e028336.

López Robles, C. W., y Rivera, D. (2013). Aproximaciones de política migratoria para Guatemala. URL. Guatemala.

Murillo Muñoz, Javier y Molero Alonzo, F. (2016). Relación entre la Satisfacción Vital y otras Variables Psicosociales de Migrantes Colombianos en Estados Unidos. Revista Colombiana de Psicología, 25(1), 15-32. Disponible en: https://dx.doi. org/10.15446/rcp.v25n1.44308.

Neto, F. (1995). Predictors of satisfaction with life among second generation migrants. Social Indicators Research, 35(1), 93-116.

Olgiati, A., Calvo, R., y Berkman, L. (2013). Are migrants going up a blind alley? Economic migration and life satisfaction around the world: Cross-national evidence from Europe, North America and Australia. Social Indicators Research, 114(2), 383-404.

OMS (2003a). Informe sobre la salud en el Mundo 2003: forjemos el futuro. Ginebra, Organización Mundial de la Salud. Disponible en: http://www.who.int/whr/2013/ report/es/.

Pérez-Escoda, N., y Alegre, A. (2014). Satisfacción con la vida: predictores y moderadores. Comunicació presentada a: I Congrés Internacional d'Educació Emocional. X Jornades d'Educació Emocional. Psicologia positiva i benestar. Barcelona, Universitat de Barcelona (Institut de Ciències de l'Educació), 2014. 978-84-697-12252. pp. 447-456.

Peterson, C., Park, N., y Seligman, M. E. (2005). Orientations to happiness and life satisfaction: The full life versus the empty life. Journal of happiness studies, 6(1), $25-41$.

Piore, M. J. (1979). Birds of Passage: Migrant Labour in Industrial Societies. Cambridge, United Kingdom: Cambridge Univer. 
Pozo, C., Hernández, S., y Alonso, E. (2004). Apoyo social y bienestar subjetivo en un colectivo de inmigrantes: ¿Efectos directos o amortiguadores? Boletín de psicología, (80), 79-96.

Programa de las Naciones Unidas para el Desarrollo (2016). Más allá del conflicto, luchas por el bienestar. Informe Nacional de Desarrollo Humano. Disponible en: http://desarrollohumano.org.gt/wp-content/uploads/2016/04/INDH_Completo_ digital-1.pdf.

Rodríguez Vignoli, J. (2004). Migración interna en América Latina y el Caribe: estudio regional del período 1980-2000.

Shin, D. C., y Johnson, D. M. (1978). Avowed happiness as an overall assessment of the quality of life. Social Indicators Research, 5, 475-492. doi: 10.1007/ BF00352944

Sosa, F., y Zubieta, E. (2012). Aculturación psicológica y creencias en estudiantes universitarios migrantes y no migrantes. Hologramática, 2(17), 45-70.

UNODC. (2013). Global study on homicide 2013. Retrieved from https://www.unodc. org

Vallejo-Martín, M., Jiménez, M., \& del Pilar, M. (2016). Satisfacción vital y su relación con otras variables psicosociales en población española residente en Alemania. Escritos de Psicología (Internet), 9(2), 12-21.

Zubieta, Elena M., y Delfino, Gisela. (2010). Satisfaction with life, psychological and social well being in college students. Anuario de investigaciones, 17, 277 283. Recuperado en 02 de mayo de 2017, de http://www.scielo.org.ar/scielo. php?script=sci_arttext\&pid=S1851-16862010000100027\&lng=es\&tlng=en . 